\title{
BMJ Global Health Protecting essential health services in low-income and middle-income countries and humanitarian settings while responding to the COVID-19 pandemic
}

Karl Blanchet (D , , ${ }^{1}$ Ala Alwan, ${ }^{2,3}$ Caroline Antoine, ${ }^{4}$ Marion Jane Cros, ${ }^{5}$ Ferozuddin Feroz, ${ }^{6}$ Tseguaneh Amsalu Guracha, ${ }^{7}$ Oystein Haaland, ${ }^{8}$ Alemayehu Hailu (D) , ${ }^{8}$ Peter Hangoma (D) , ${ }^{9}$ Dean Jamison, ${ }^{10}$ Solomon Tessema Memirie, ${ }^{11,12}$ Ingrid Miljeteig (D) , ${ }^{13,14}$ Ahmad Jan Naeem, ${ }^{6}$ Sara L. Nam, ${ }^{15}$ Ole Frithjof Norheim, ${ }^{8}$ Stéphane Verguet (D) , ${ }^{16}$ David Watkins (D) , ${ }^{3}$ Kjell Arne Johansson (1) ${ }^{17}$

To cite: Blanchet K, Alwan A, Antoine $\mathrm{C}$, et al. Protecting essential health services in low-income and middleincome countries and humanitarian settings while responding to the COVID-19 pandemic. BMJ Global Health 2020;5:e003675. doi:10.1136/ bmjgh-2020-003675

Handling editor Seye Abimbola

Received 10 August 2020

Revised 14 September 2020

Accepted 16 September 2020

\section{ABSTRACT}

In health outcomes terms, the poorest countries stand to lose the most from these disruptions. In this paper, we make the case for a rational approach to public sector health spending and decision making during and in the early recovery phase of the COVID-19 pandemic. Based on ethics and equity principles, it is crucial to ensure that patients not infected by COVID-19 continue to get access to healthcare and that the services they need continue to be resourced. We present a list of 120 essential nonCOVID-19 health interventions that were adapted from the model health benefit packages developed by the Disease Control Priorities project.

\section{INTRODUCTION}

Evidence is accumulating that the COVID-19 pandemic is creating unprecedented disruptions in the delivery of routine health services in many countries of the world. Compounding this problem, economic fallout generated by lockdown policies is putting pressure on Ministries of Health to cut public spending or divert resources to the COVID-19 response and thus compromising other essential and even life-saving non-COVID-19 services. In health outcomes terms, the poorest countries stand to lose the most from these disruptions. ${ }^{12}$ In this paper, we make the case for a rational approach to public sector health spending and decision making during and in the early recovery phase of the COVID-19 pandemic. Based on ethics and equity principles, it is crucial to ensure that patients non infected by COVID-19 continue to get access
Summary box

COVID-19 creates unprecedented disruptions in delivery of routine healthcare.

- It is crucial to ensure continued access to essential non-COVID-19 healthcare.

- A concrete list of 120 essential non-COVID-19 health interventions has been developed based on the Disease Control Priorities-3 highest priority package (HPP).

- Adjustments of HPP was made based on level of urgency of interventions and contextual factors.

- The adjusted HPP could be used by governments and donors as input for discussions about disinvestments and continued investments during the COVID-19 pandemic.

to healthcare and that the services they need continue to be resourced. We present a list of 120 essential non-COVID-19 health interventions that were adapted from the model health benefit packages developed by the Disease Control Priorities (DCP) project. ${ }^{3}$ These 120 interventions underwent careful scrutiny and were selected in part based on the probable magnitude of the harms that would occur from interruptions or disinvestments. We argue that the selected interventions are the most essential to deliver and protect, even if substantial resources need to be diverted to the COVID-19 response. Even if it has previously been shown that continued scale-up of all of these interventions are important for countries to achieve the health Sustainable Development Goals (SDG) targets, especially 
SDG target 3.8, our scope is more modest. Here, focusing on routine healthcare services, we provide concrete guidance on which interventions policy-makers and donors need to protect from disinvestments. Our model list of interventions can serve as normative guidance to governments and humanitarian agencies working to define national and local guidance for protecting essential routine services in low-income and middle-income countries and humanitarian settings.

\section{THE NEED TO PROTECT ESSENTIAL HEALTH SERVICES}

Governments in low-income and middle-income countries and relief agencies need to make clear decisions to not only mitigate the impact of the COVID-19 pandemic but also deliver essential routine services to their populations. This is a clear message from WHO in their operational guidance for maintaining essential health services during an outbreak. ${ }^{4}$ While each country will need to define essential services according to their epidemiological profile, health system capacity and available resources, we believe that guidance on the type of essential services required by low-income and lower-middleincome countries is a valuable contribution to inform urgent decision making during health crises.

Since March 2020, routine immunisation services have been disrupted on a global scale putting millions of children at risk of diseases like diphtheria, measles and polio. According to data collected by the WHO, UNICEF, Gavi and the Sabin Institute is likely to affect around 80 million children under 1 year worldwide. Some countries have reported the emergence of new outbreaks of cholera, measles and Ebola, health personnel being absent from facilities because of quarantine, lack of personal protective equipment or fear, and that patients are not seeking care because of perceived risk of infectious spread and the consequences of lockdown (eg, travel restrictions, closure of markets, decrease in income).$^{5}$ An important concern is that decline in supply and demand for nonCOVID-19 essential routine health services may exacerbate the general health situation and lead to excess mortality beyond what is directly attributed to the pandemic. Studies indicated that excess mortality superseded Ebola deaths during the 2014-2015 outbreak in West-Africa. ${ }^{67}$

The selected 120 essential health interventions that should be unconditionally protected and delivered despite the disruptions caused by COVID-19 were extracted from the highest priority package (HPP) for universal health coverage (UHC) developed by the DCP project. The HPP list of interventions was used as a starting point for discussions and adaptation together with national policymakers in Afghanistan, ${ }^{8}$ Ethiopia, Pakistan and Zanzibar. All invited policy-makers in each of these countries had past experience with translating the evidence from the original list of HPP interventions into national health benefit packages, providing an important source of information from diverse contexts. Given substantial resource scarcity, we present a modified highest priority model list of essential services that are urgent for patients and provide the greatest health impact given resource scarcity. Subject to local disease burden and circumstances, access to these services should be protected for all residents irrespective of income, refugee or migrant status, gender and place of residence.

In countries where the response to the COVID-19 pandemic leads to substantial limitations of resources, the scarcity of health services will affect healthcare seeking behaviour and all patients' health, including those with life-threatening conditions requiring prompt medical attention. Fair allocation of resources that prioritises the value of maximising benefits applies across all patients who need healthcare. There should be no difference in allocating scarce resources between patients with COVID-19 and those with other equally serious medical conditions.

\section{OBJECTIVES OF THE PRIORITISATION PROCESS}

Service providers and decision-makers are now amidst processes aiming to identify which essential services to protect, identify areas where resources can be reallocated to the COVID-19 response, mitigate the effect of the COVID-19 pandemic on the effectiveness of routine services, and restore trust of the public vis-à-vis health services.

Beyond the specific response to the COVID-19 pandemic, decisions need to be made about allocation of the limited resources between continuation of routine services, adjustment of routine services and postponement of non-essential services. Decisions will also need to be made on shifting the platform of delivery of some interventions based on health system capacity (eg, shifting some interventions from community to health centre considering the level of workload of community health workers in contract tracing).

\section{CRITERIA AND PROCESS FOR SELECTING INTERVENTIONS}

These further prioritisation decisions need to be made based on evidence and transparent selection criteria on fair priority setting widely accepted by policy-makers, practitioners and academics, such as impact on mortality and morbidity, urgency (ie, impact on patient health of delaying services), cost-effectiveness, protection of politically sensitive interventions, financial risk protection and public acceptability. The members of the global and country DCP teams, coauthors of this paper were consulted through group meetings and online tools to comment on the essential list of health services.

Standard principles for selection are based on humanitarian and UHC principles ${ }^{9-11}$ :

- Treating people equally (non-discrimination).

- Maximising the benefits produced by scarce resources (saving the most individual lives or saving the most life-years by giving priority to patients likely to survive longest after treatment). 
- Giving priority to the worst off (in terms of poverty or in terms of health: the sickest or those who will have lived the shortest lives if they die untreated).

These principles can be combined with other goals and principles relevant for governments and agencies (eg, the humanitarian principles of humanity, impartiality and neutrality).

Our recommendations first emerged from the 115 HPP interventions proposed by DCP3 in $2018 .{ }^{12}$ Originally, interventions in HPP were identified after wide consultations considering evidence on burden of disease, implementation feasibility, and value for money. ${ }^{3}$ Value for money includes considerations of cost-effectiveness, priority to the worst off and financial risk protection. We modified the original HPP and added three considerations of particular relevance under the present circumstances:

1. Context-specific relevance (revisions made by national policy makers in Afghanistan, Ethiopia, Pakistan and Zanzibar).

2. Urgency (for patient) (high-impact interventions for which delays would substantially increase mortality and morbidity).

3. Non-urgency (important services where delayed provision (3-6 months) would not affect the health impact).

The original HPP list was informed by wide consultations and actual data and analysis. However, this revised list was informed by extensive deliberations on how contextual factors and urgency (1-3 above) could justify inclusion or exclusion, or revision of delivery platform, of each HPP intervention. Country DCP teams from Afghanistan, Ethiopia, Pakistan and Zanzibar were included in this COVID-19 revision of the HPP because all of these countries have experience with using the DCP3 framework and the HPP in detailed revisions of national essential healthcare packages. Even though they represent diverse settings, all low-income and middle-income settings are not represented here. Implementation of the revised HPP list therefore needs to be adapted to context and resources available.

\section{SCOPE OF THE PRIORITY LIST OF ESSENTIAL SERVICES}

The priority list of 120 essential services is mainly designed for low-income and middle-income countries and humanitarian settings. For countries not hard hit by COVID-19, the full range of HPP health services is still relevant, even if not fully implemented in every country for reasons of resource constraints.

Table 1 provides the proposed list of essential services within each programme area. Only a few interventions from the original HPP list were not included in the current revised list. The five interventions that should be postponed during the time of COVID-19 are: (1) Mass media messages concerning healthy eating or physical activity; (2) Management of osteomyelitis, including surgical debridement for refractory cases; (3) Cataract extraction and insertion of intraocular lens; (4) Elective surgical repair of common orthopaedic injuries (eg, meniscal and ligamentous tears) in individuals with severe functional limitation and (5) Repair of cleft lip and cleft palate.

We have conducted a minor revision of the original HPP because most of the HPP interventions have high levels of urgency. An immediate interruption of these services, due to COVID-19 disinvestments, may have serious negative impact on individual patients and population health. Immediate interruption of any of the, for example, emergency care interventions, obstetric or neonatal interventions, surgery interventions or mental healthcare interventions will most likely worsen the prognosis for all patients currently receiving this type of care (or patients that would receive this type of care if there were no COVID-19 pandemic). Therefore, and since all the original HPP interventions are best buys to begin with, it is hard to justify a substantial reduction in number of interventions to protect from disinvestments.

In order to protect patients and community health workers, and considering the additional workload of community health workers busy in COVID-19 contact tracing and surveillance, several HPP interventions were shifted from community to health centre level: Postgenderbased violence care, including counselling, provision of emergency contraception, and rape-response referral (medical and judicial); iron and folic acid supplementation to pregnant women and adolescent girls; provision of food or caloric supplementation to pregnant women in food insecure households; identify and refer patients with high risk, including pregnant women, young children and those with underlying medical conditions. COVID-19 presents an opportunity to introduce digital tools in delivery of healthcare. Tools like telemedicine, mobile consultations or digital consultations may serve as useful supplements to existing delivery platforms, as documented on the COVID-19 humanitarian platform. ${ }^{13}$ Digital or mobile consultations still need to be anchored within existing delivery platforms at the community, health centre or hospital level.

Coverage of the remaining essential services should be, at least, unchanged during the COVID-19 pandemic and still provided to patients irrespective of income, refugee or migrant status, gender and place of residence. These services must still be subsidised by domestic and external funding as much as possible. The promotive, preventive, curative and rehabilitative interventions included in the priority package are considered the minimum that people can expect to receive through the various healthcare delivery mechanisms and facilities available at various levels of the health system (community, health centre and hospital levels (first level and referral hospitals)). Countries where these interventions are either not available or have low coverage should strive to deliver them, and in countries where they are already implemented, they should be maintained and protected during times of pandemics. The public should be informed, through public media campaigns, that these services will 
Table 1 Programme areas and examples of essential routine services per delivery platform to be unconditionally protected during the COVID-19 pandemic

\begin{tabular}{|c|c|c|}
\hline Programme & Interventions & Delivery platform* \\
\hline \multicolumn{3}{|c|}{ Sexual and reproductive health } \\
\hline & Provision of condoms and hormonal contraceptives & Health centre \\
\hline & Modern contraceptives of client choice, long lasting & First-level hospital \\
\hline & Medical abortion & Health centre \\
\hline & Surgical abortion & First-level hospital \\
\hline & $\begin{array}{l}\text { Postgender-based violence care, including counselling, provision of emergency } \\
\text { contraception, and rape-response referral (medical and judicial) }\end{array}$ & Health centre \\
\hline
\end{tabular}

Maternal and newborn health

\section{Antenatal care}

Early detection and management of syphilis, hypertension, pre-eclampsia, diabetes and other pregnancy complications

Detection and treatment of bacteriuria

Tetanus immunisation

\section{Basic emergency obstetric care}

Assisted vaginal delivery (including vacuum extraction)

Administering antibiotics, uterotonic drugs oxytocin and anticonvulsants (magnesium sulphate)

Manual removal of the placenta

Removal of retained products following miscarriage or abortion

\section{Comprehensive emergency obstetric care}

Surgery (eg, caesarean sections, hysterectomy)

Safe blood transfusion

Forceps extraction, if properly trained
Health centre

Health centre

Health centre

Health centre

Health centre

Health centre

Health centre

Health centre

Health centre

First-level hospital

First-level hospital

First-level hospital

First-level hospital

Antenatal corticosteroid for preterm labour, including early detection and referral at First-level hospital health centres

Induction of labour (beyond 41 weeks)

Management of pregnancy induced hypertension, including pre-eclampsia/ eclampsia

Ectopic pregnancy case management

Management of maternal sepsis

\section{Basic neonatal care}

Basic neonatal resuscitation care (with bag and mask)

Thermal protection for all babies, especially preterms

Hygienic cord care

Kangaroo mother care and additonal feeding support (eg, with nasogastric tube/ cup feeding) for small preterm babies

\section{Comprehensive neonatal care}

Management of newborn complications, neonatal meningitis and other very serious infections

Neonatal acute respiratory infection detection and treatment (intravenous antibiotics, oxygen therapy and respiratory support)

Newborn sepsis-injectable antibiotics

Management of jaundice
First-level hospital

First-level hospital

First-level hospital

First-level hospital

Health centre

Health centre

Health centre

Health centre

Health centre

First-level hospital

First-level hospital

First-level hospital

First-level hospital

First-level hospital 
Table 1 Continued

\begin{tabular}{lll}
\hline Programme Interventions Delivery platform* & Deras
\end{tabular}

Routine childhood vaccines (diptheria, pertussis, tetanus, polio, Bacillus Calmette- Community guerin (BCG), measles, hepatitis $B$, Hib, rubella)

Pneumococcus vaccination

Community

Rotavirus vaccination

Community

Tetanus toxoid immunisation among schoolchildren

Community

Integrated community case management of childhood illness

Community

Integrated management of childhood illness

Health centre

Full supportive care for severe childhood infections

First-level hospital

HIV and sexually transmitted infections (STIs)

Community-based HIV education and testing services

Community

Provision of condoms to at risk populations

Community

Cotrimoxazole prophylaxis

Community

HIV treatment

Health centre

Provider HIV, STI, Hepatitits testing and linkage to care

Health centre

Prevention of mother to child HIV transmission (option $\mathrm{B}+$ ) and syphilis

Health centre

Antiretrovirals for tuberculosis (TB)/HIV co-infection

Health centre

Syndromic management of sexually transmitted infections

Health centre

Malaria

Indoor residual spraying in high endemic settings

Community

Insecticide-treated bednets for pregnant women and children

Community

Malaria treatment with artemisinin-based combination therapy preceded by rapid Community diagnostic tests if feasible

Malaria chemoprophylaxis in high endemic season (p. falciparum dominant)

Community

Intermittent malaria prevention in infancy

Community

Intermittent malaria prevention during pregnancy

Community

Comprehensive management of severe malaria

First-level hospital

TB

Active case finding followed by treatment when needed in HIV +individuals and

Population based other high-risk groups

TB, contact tracing

Community

TB diagnosis and treatment (including extrapulmonary)

Health centre

Referral of cases of treatment failure for drug susceptibility testing; enrollment of those with multidrug resistant TB for treatment per WHO guidelines (either short or

First-level hospital long regimen)

Neglected tropical diseases

Sustained vector management for chagas disease, visceral leishmaniasis, dengue, Population based and other nationally important causes of nonmalarial fever

Mass drug administration for lymphatic filariasis, onchocerciasis, schistosomiasis, Community soil-transmitted helminthiases and trachoma, and foodborne trematode infections

Early detection and treatment of Chagas disease, human African trypanosomiasis, First-level hospital leprosy, and leishmaniases

Infections in general

Continued 
Table 1 Continued

\begin{tabular}{|c|c|c|}
\hline Programme & Interventions & Delivery platform* \\
\hline & Pharyngitis treatment & Health centre \\
\hline & $\begin{array}{l}\text { Fever evaluation and basic management, clinically stable, WHO Integrated } \\
\text { Management of Adolescent and Adult Illness/Integrated Management of } \\
\text { Childhood Illness (IMAI) guidelines, with referral of unstable individuals }\end{array}$ & Health centre \\
\hline & $\begin{array}{l}\text { Fever evaluation and comprehensive management, clinically unstable, WHO IMAI } \\
\text { guidelines }\end{array}$ & First-level hospital \\
\hline & Refractory febrile illness including etiologic diagnosis & Referral hospital \\
\hline \multicolumn{3}{|l|}{ Cancer } \\
\hline & Human Papilloma virus vaccine & Community \\
\hline & Early detection of cancer symptoms & Health centre \\
\hline & Early detection and treatment of early-stage cervical cancer & Referral hospital \\
\hline & $\begin{array}{l}\text { Treatment of early stage breast cancer, multimodal approaches (including generic } \\
\text { chemotherapy), curative intent }\end{array}$ & Referral hospital \\
\hline & $\begin{array}{l}\text { Treatment of early-stage colorectal cancer, multimodal approaches (including } \\
\text { generic chemotherapy), curative intent }\end{array}$ & Referral hospital \\
\hline & $\begin{array}{l}\text { Treatment of early-stage childhood cancers (Burkitt and Hodgkin lymphoma, acute } \\
\text { lymphoblastic leukaemia, retinoblastoma, Wilms tumour), curative intent }\end{array}$ & Referral hospital \\
\hline
\end{tabular}

Cardiovascular and related disorders (metabolic disorders, kidney failure, etc)

Cardiovascular disease (CVD), primary prevention with absolute risk approach Health centre (antihypertensives, statins)

CVD, secondary prevention (aspirin, beta blockers, ACE inhibitors, statins) Health centre

Secondary prophylaxis for rheumatic fever or established rheumatic heart disease, Health centre penicillin

Active case finding and management of diabetes (glycaemic control, Health centre antihypertensives, statins, and consistent foot care)

Management of heart failure (diuretics, beta-blockers, ACE inhibitors, and Health centre mineralocorticoid antagonists)

Management of acute heart failure

First-level hospital

Aspirin for all cases of suspected acute myocardial infarction

First-level/referral hospital

Mental health disorders

Active case finding of psychosis, depression, anxiety, bipolar disorder and post- Health centre traumatic stress disorder (PTSD)

Management of depression and anxiety

Health centre

Management of PTSD

Health centre

Management of bipolar disorder

Health centre

Management of psychosis (schizophrenia)

Health centre

Management for attention deficit hyperactivity disorder

Health centre

Basic psychosocial follow-up for suicide and self harm

Health centre

\begin{tabular}{|lc|}
$\begin{array}{l}\text { Substance use disorders } \\
\text { Opioid agonist treatment and safe needles }\end{array}$ & Health centre \\
$\begin{array}{l}\text { Neurological disorders } \\
\text { Epilepsy treatment }\end{array}$ & Health centre \\
\hline $\begin{array}{l}\text { Musculoskeletal disorders } \\
\text { Combination therapy for moderate to severe rheumatoid arthritis, low-dose } \\
\text { corticosteroids, folic acid supplementation, disease-modifying anti-rheumatic } \\
\text { drugs (including methotrexate) }\end{array}$ & First-level hospital \\
\hline Surgery & \\
\hline
\end{tabular}

Continued 


\begin{tabular}{|c|c|c|}
\hline \multirow[t]{10}{*}{ Programme } & Interventions & Delivery platform* \\
\hline & Drainage of abscess & Health centre \\
\hline & Drainage of dental abscess & Health centre \\
\hline & Management of bowel obstruction & First-level hospital \\
\hline & Appendectomy & First-level hospital \\
\hline & Colostomy & First-level hospital \\
\hline & Hernia repair & First-level hospital \\
\hline & Management of osteomyelitis & First-level hospital \\
\hline & Repair of peptic ulcer perforations & First-level hospital \\
\hline & Urinary catheterisation/suprapubic cystostomy & First-level hospital \\
\hline \multicolumn{3}{|c|}{ Emergency care } \\
\hline & First aid & Community \\
\hline & Basic life support and first aid for burns, bleeding and wounds and choking & Community \\
\hline & Basic emergency care & Health centre \\
\hline & Management of non-displaced fractures & Health centre \\
\hline & Resuscitation with basic life support measures & Health centre \\
\hline & $\begin{array}{l}\text { Identify and refer patients with high risk including pregnant women, young } \\
\text { children, and those with underlying medical conditions }\end{array}$ & Health centre \\
\hline & \multicolumn{2}{|l|}{ Advanced emergency care } \\
\hline & Suturing laceration & Health centre \\
\hline & Traction for fractures & First-level hospital \\
\hline & Irrigation and debridement of open fractures & First-level hospital \\
\hline & Resuscitation with advanced life support measures & First-level hospital \\
\hline & Trauma laparotomy & First-level hospital \\
\hline & Trauma-related amputations & First-level hospital \\
\hline & Tube thoracostomy & First-level hospital \\
\hline & Management of septic arthritis & First-level hospital \\
\hline & Urgent orthopaedic management of injuries & Referral hospital \\
\hline \multicolumn{3}{|c|}{ Palliative care and pain control } \\
\hline & Palliative care and pain control & Health centre \\
\hline & Prevention/relief of refractory suffering and of acute pain & First-level hospital \\
\hline \multicolumn{3}{|l|}{ Nutrition } \\
\hline & Detection and referral of severe acute malnutrition & Community \\
\hline & Vit. A and Zinc to children and food for women & Community \\
\hline & $\begin{array}{l}\text { Iron and folic acid supplementation, pregnant women, adolescent girls. Provision } \\
\text { of food or caloric supplementation to pregnant women in food insecure } \\
\text { households }\end{array}$ & Health centre \\
\hline & Promotion of early and exclusive breastfeeding or complementary feeding & Health centre \\
\hline & $\begin{array}{l}\text { Treatment of severe acute malnutrition for cases presenting with or without } \\
\text { associated medical complications (eg, Infections) }\end{array}$ & $\begin{array}{l}\text { Health centre and first- } \\
\text { level hospital }\end{array}$ \\
\hline \multicolumn{3}{|c|}{ Water supply, sanitation and hygiene } \\
\hline & & Continued \\
\hline
\end{tabular}


Table 1 Continued

\begin{tabular}{lll}
\hline Programme & Interventions & Delivery platform* $^{*}$ \\
\hline & $\begin{array}{l}\text { WASH: establish quality WASH facilities in schools, workplaces, public spaces, } \\
\text { and healthcare facilities }\end{array}$ & Population based \\
WASH: targeted WASH subsidies to poor and vulnerable groups & Population based \\
WASH: enact national standards for safe drinking water and sanitation within and & Population based \\
outside households and institutions & Community \\
& Media messages on handwashing and air pollution & Community \\
& WASH behavioural change interventions, such as community-led total sanitation & \\
Health education and behavioural change communication & All facilities \\
\hline Health system services & Laboratory services
\end{tabular}

*The delivery platform will vary by country. We suggest here the recommended lowest delivery platform.

be offered in a safe manner, if necessary, in designated locations, free of charge and with acceptable quality.

\section{PROCESS AND IMPLEMENTATION}

We propose that governments and agencies that are in the process of defining which essential services should be protected under the COVID-19 crisis use our model list as input for further deliberation with key stakeholders, citizens, funders, local and national decision-makers. Local context may allow for a larger set of services to be provided. International organisations may also adapt the list through a broader, more representative process. We expect that the COVID-19 pandemic will affect the share of domestic resources invested in total health expenditure, considering that economic growth is the main driver for domestic resources for health. This list of priority essential interventions may also become an important source of guidance for the post-COVID-19 period.

\footnotetext{
Author affiliations

${ }^{1}$ Geneva Centre of Humanitarian Studies, University of Geneva Faculty of Medicine, Geneve, Switzerland

${ }^{2}$ Faculty of Public Health and Policy, London School of Hygiene \& Tropical Medicine, London, UK

${ }^{3}$ University of Washington, Seattle, Washington, USA

${ }^{4}$ Action Contre la Faim, Paris, France

${ }^{5}$ Global Financing Facility/World Bank, Addis Ababa, Ethiopia

${ }^{6}$ Ministry of Public Health, Kabul, Afghanistan

${ }^{7}$ Global Financing Facility/World Bank, Addis Abab, Ethiopia

${ }^{8}$ Department of Global Public Health and Primary Care, University of Bergen, Bergen, Norway

${ }^{9}$ Health Policy and Management, University of Zambia, Lusaka, Zambia

${ }^{10} \mathrm{Global}$ Health Sciences, University of California, San Francisco, California, USA

${ }^{11}$ Department of Global Public Health and Primary Care, Universitetet i Bergen, Bergen, Norway

${ }^{12}$ Department of Global Health and Population, Harvard T.H. Chan School of Public Health, Cambridge, Massachusetts, USA

${ }^{13}$ Department of Global Health and Primary Health Care, University of Bergen Faculty of Medicine and Dentistry, Bergen, Norway

${ }^{14}$ Department of Research and Development, Haukeland University Hospital, Bergen, Norway

${ }^{15}$ Options Consultancy Services Ltd, London, UK
}

${ }^{16}$ Global Health and Population, Harvard University T H Chan School of Public Health, Boston, Massachusetts, USA

${ }^{17}$ Department of Global Public Health and Primary care, University of Bergen Faculty of Medicine and Dentistry, Bergen, Norway

Twitter Karl Blanchet @BlanchetKarl, Alemayehu Hailu @alemayehu4u2, Peter Hangoma @HangomaPeter, David Watkins @davidawatkins and Kjell Arne Johansson @KA_Johansson

Contributors $\mathrm{KB}, \mathrm{OFN}$, DW and KAJ designed the prioritisation process, drafted the various versions of the list of interventions and the paper. AJN, AH, CA, FF, IM, MJC, OH, PH, STM, SN and TAG reviewed the first list of interventions and reviewed the final list and the draft and final paper. AA, DJ and SV reviewed the final version of the list of interventions and the draft and final paper.

Funding The authors have not declared a specific grant for this research from any funding agency in the public, commercial or not-for-profit sectors.

Competing interests None declared.

Patient consent for publication Not required.

Provenance and peer review Not commissioned; externally peer reviewed.

Data availability statement All data relevant to the study are included in the article.

Open access This is an open access article distributed in accordance with the Creative Commons Attribution Non Commercial (CC BY-NC 4.0) license, which permits others to distribute, remix, adapt, build upon this work non-commercially, and license their derivative works on different terms, provided the original work is properly cited, appropriate credit is given, any changes made indicated, and the use is non-commercial. See: http://creativecommons.org/licenses/by-nc/4.0/.

\section{ORCID iDs}

Karl Blanchet http://orcid.org/0000-0003-0498-8020

Alemayehu Hailu http://orcid.org/0000-0003-4872-8036

Peter Hangoma http://orcid.org/0000-0002-6573-5628

Ingrid Miljeteig http://orcid.org/0000-0001-5738-017X

Stéphane Verguet http://orcid.org/0000-0003-4128-0849

David Watkins http://orcid.org/0000-0001-6341-9595

Kjell Arne Johansson http://orcid.org/0000-0001-8912-8710

\section{REFERENCES}

1 Graham WJ, Afolabi B, Benova L, et al. Protecting hard-won gains for mothers and newborns in low-income and middle-income countries in the face of COVID-19: call for a service safety net. BMJ Glob Health 2020;5:e002754.

2 Roberton T, Carter ED, Chou VB, et al. Early estimates of the indirect effects of the COVID-19 pandemic on maternal and child mortality in low-income and middle-income countries: a modelling study. Lancet Glob Health 2020;8:e901-8. 
3 Jamison DT, Alwan A, Mock CN, et al. Universal health coverage and intersectoral action for health: key messages from disease control priorities, 3rd edition. Lancet 2018;391:1108-20.

4 World Health Organisation. Maintaining essential health services: operational guidance for the COVID-19 context. Geneva World Health Organisation; 2020.

5 World Health Organisation, American Red Cross U.S, CDC, UNICEF, UN Foundation. More than 117 million children at risk of missing out on measles vaccines, as COVID-19 surges. Geneva; 2020

6 Parpia AS, Ndeffo-Mbah ML, Wenzel NS, et al. Effects of response to 2014-2015 Ebola outbreak on deaths from malaria, HIV/AIDS, and tuberculosis, West Africa. Emerg Infect Dis 2016;22:433-41.

7 Walker PGT, White MT, Griffin JT, et al. Malaria morbidity and mortality in Ebola-affected countries caused by decreased healthcare capacity, and the potential effect of mitigation strategies: a modelling analysis. Lancet Infect Dis 2015;15:825-32.
8 Blanchet K, Ferozuddin F, Naeem AJJ, et al. Priority setting in a context of insecurity, epidemiological transition and low financial risk protection, Afghanistan. Bull World Health Organ 2019;97:374-6.

9 Emanuel EJ, Persad G, Upshur R, et al. Fair allocation of scarce medical resources in the time of Covid-19. N Engl J Med 2020;382:2049-55

10 UNOCHA. The humanitarian principles. New York: UNOCHA, 2012.

11 World Health Organization. Making fair choices on the path to universal health coverage. Geneva; 2014.

12 Watkins DA, Qi J, Kawakatsu Y, et al. Resource requirements for essential universal health coverage: a modelling study based on findings from disease control priorities, 3rd edition. Lancet Glob Health 2020;8:e829-39.

13 Geneva Centre of Humanitarian Studies, Johns Hopkins Bloomberg School of Public Health, London School of Hygiene and Tropical Medicine. COVID-19 humanitarian platform, 2020. Available: https:// www.covid19humanitarian.com/ 